\title{
Thermo-mechanical fatigue test of a wrought Co-based alloy as potential tooling material for die casting
}

\author{
Ning Tang ${ }^{a}$, Yunping Li ${ }^{a},{ }^{*}$, Phacharaphon Tunthawiroon ${ }^{b}$, Yuichiro Koizumi ${ }^{a}$, \\ Akihiko Chiba $^{\text {a }}$ \\ ${ }^{a}$ Institute for Materials Research, Tohoku University, Sendai 980-8577, Japan \\ ${ }^{\mathrm{b}}$ Graduate School of Engineering, Tohoku University, Sendai 980-8577, Japan \\ *Corresponding authors: lyping@imr.tohoku.ac.jp
}

\begin{abstract}
Thermo-mechanical fatigue (TMF) test of a wrought Co-Cr-Mo alloy was investigated, and Co-based alloy showed superior TMF properties than the control sample of a hot work tool steel. During TMF test, high oxidation resistance and increased hardness were observed in the Co-based alloy in contrast to the low oxidation resistance and decreased hardness in the tool steel. The y-phase-to- $\varepsilon$-phase transformation of the Co-based alloy was considered as the main hardening factor during the TMF test.
\end{abstract}

Keyword: Thermo-mechanical fatigue (TMF); Co-based alloy; EBSD; Oxidation; Hardness measurement; Phase transformation 


\section{Introduction}

Die casting is a near net-shaped process that has been widely applied to produce the non-ferrous alloys (such as $\mathrm{Al}, \mathrm{Mg}, \mathrm{Cu}$, and $\mathrm{Zn}$ based alloys) with high strength, close tolerance, complex shape, various surface finishing, and high manufacturing rate [1-3]. However, the high efficiency of this casting technique has to be guaranteed by the high performance of molds (called dies) to withstand the severe conditions in service. The elevated temperature, wear, molten metal corrosion and the thermo-mechanical fatigue (TMF, induced by the cyclic thermomechanical loading during die casting cycles) are the major threats to the durability of molds. Surface of molds would be gradually destroyed by these attacks, giving rise to both the decrease in quality of cast parts and the early failure of molds. Currently, hot work tool steels are mainly adopted as tooling materials for die casting due to their superior mechanical properties; however, their low resistance to molten metal corrosion and TMF cracking are the urgent problems having to be resolved in the die casting industry [4-7]. Various techniques such as surface treatments and coatings have been applied to tool steels with the purpose of enhancing the resistance to wear and molten metal corrosion [8-10]; however the TMF properties were significantly deteriorated by the surface treatments $[11,12]$. Since tooling accounts for a great part of the total cost of the final die casting products, the tooling materials with longer service life are extremely effective in reducing the manufacturing cost in die casting industry. 
On the other hand, Co-based alloys have been widely applied to withstand severe conditions for more than a century, because of their excellent wear resistance, heat resistance and corrosion resistance [13-15]. Recently, by virtue of some unique properties as compared with tool steels, Co-based alloys have been considered to be the promising tooling materials for die casting. Mihelich et al. [16] reported that $\mathrm{Co}-\mathrm{Cr}$ alloys showed much higher corrosion/erosion resistance to molten Al alloy than both tool steels and Ni-based alloys. Quite recently, aiming at developing novel high temperature tooling materials for the thixoforming (a forming technology similar to die casting) of Al alloys, Mg alloys and steels, a series of works were carried out by Birol [17-19] on the thermal fatigue tests of Co-Cr alloys, Ni-based alloys and tool steels. The results indicated that Co-Cr alloys showed the highest resistance to the thermal fatigue cracking among the tested alloys and this high performance was ascribed to their high resistance to both oxidation and temper softening [17, 18]. Especially, the compact oxide film, which formed on the surface of $\mathrm{Co}-\mathrm{Cr}$ alloys, not only enhanced the resistance to oxidation and thermal fatigue cracking as mentioned above [17, 18], but also significantly improved the resistance to molten Al corrosion according to our previous researches $[20,21]$. Therefore, by virtue of their superior resistance to thermal fatigue cracking and molten metal corrosion as compared with the currently used tool steels, Co-based alloys are considered as the prospective tooling materials in die casting and the other industry with similar service environments. 
Nevertheless, very few researches were reported on the TMF tests of Co-based alloys. Birol [17-19] well revealed the superior thermal fatigue properties of Cobased alloys; however, the test system was inadequate to simulate the service environments of the die casting tools due to that the cyclic mechanical loading was not taken into account. In addition, Birol mainly investigated the thermal fatigue behavior of Co-based alloy with typical cast structure, and so far, there is no report on the TMF tests of the wrought Co-based alloys, even though their properties were expected to be higher than the corresponding cast alloys owing to the modified microstructure. In this study, for the development of a novel Cobased tooling material for die casting, the TMF test of a wrought $\mathrm{Co}-\mathrm{Cr}-\mathrm{Mo}$ alloy was carried out under programmed cycles of both temperature and mechanical strain, and the superior TMF life of this Co-based alloy was presented with the comparison of the tool steel for the first time.

\section{Materials and experiments}

A wrought Co-28Cr-6Mo alloy (Ф $22 \mathrm{~mm}$, supplied by Eiwa Co., Ltd., Japan) was adopted for TMF test with the comparison of the SKD61 tool steel $(\Phi 19 \mathrm{~mm}$, supplied by Daido Steel Co., Ltd., Japan). Their chemical compositions were tabulated in Table 1. For TMF tests, the bars of the test materials were machined into the tubular specimen with the geometry as show in Fig. 1. The TMF tests were carried out with Instron 8862 tester (Fig. 2). During TMF tests, the specimen was heated with an external induction coil and internal cooled with compressed air. An R-type thermocouple and a strain gauge were applied to monitor the 
temperature and strain, respectively. The conditions of TMF test were set as an out-of-phase program according to die casting cycle in industry (Fig. 3). The temperature $\left(400\right.$ to $\left.700{ }^{\circ} \mathrm{C}\right)$ and the mechanical strain $(-0.1$ to $0.1 \%)$ changed linearly in the program (32 s/cycle), and the maximum temperature coincided to the minimum mechanical strain. Especially, the total strain $\left(\varepsilon_{t}\right.$, measured by the strain gauge), thermal strain $\left(\varepsilon_{\text {th }}\right)$, and mechanical strain $\left(\varepsilon_{\text {mech }}\right.$, set in the controlling program) could be expressed by the following equation

$$
\varepsilon_{\mathrm{t}}=\varepsilon_{\mathrm{th}}+\varepsilon_{\mathrm{mech}}
$$

Before the main test, there was a pre-test, during which the total strain was adjusted to zero at the midpoint of the temperature range under zero loading and the thermal strain profile of the specimen in the test temperature range was measured. During the main test stage, the stress range of each cycle (as shown in Fig. 4) was recorded by the controlling computer automatically.

The microstructures of the Co-Cr-Mo alloy before TMF test and after failure were observed with electron backscatter diffraction (EBSD) under a fieldemission scanning electron microscope (FEI XL30S-FEG) equipped with a TSL OIM system. The image quality (IQ) map, phase map and kernel average misorientation (KAM) map were obtained with the OIM software to show the evolution of microstructures. The KAM was calculated using the average misorientation of a given pixel with its first neighbors, excluding the misorientations greater than $5^{\circ}$. The value of $\mathrm{KAM}$ evaluates the local lattice rotation of the given point, and the higher value means the higher lattice strain. 
The samples for EBSD observation were ground with $\mathrm{SiC}$ abrasive paper until 1500 grade, then polished with 1 and $0.3 \mu \mathrm{m}$ alpha alumina suspension (AP-A suspension, Struers) in sequence, and finally polished with colloidal silica suspension (OP-U suspension, Struers). Since the oxidation and spallation of oxide scale would consume the alloy matrix, the thickness of the specimens after TMF failure was measured on the cross section and then the thickness loss and its rate was calculated to reflect the oxidation resistance of the different materials under the condition of TMF tests. Moreover, the Vickers hardness of the tested materials before TMF tests and after TMF failure was evaluated on the mirrorpolished cross section with a Shimadzu HMV Micro-hardness Tester under a load of $0.5 \mathrm{Kg}$.

\section{Results and discussion}

The initial microstructure of Co-Cr-Mo alloy is shown as EBSD maps in Fig. 5. Both IQ map (Fig. 5a) and the boundaries in Fig. 5b and c show that plenty of annealing twins exist in the nearly equiaxed grains, and the average grain size was calculated as $12.1 \mu \mathrm{m}$. A nearly full $\gamma$ phase is demonstrated from the phase map (Fig $5 b$ ), and the low value of KAM indicates the residual stress is low and almost homogeneously distributed throughout the sample (Fig. 5c).

The cyclic response curves of stress range as a function of cyclic number are shown as Fig. 6. The curves of both Co-Cr-Mo alloy and tool steel almost keep constant throughout the TMF tests except for the sharp decline before fracture. 
However, the superior TMF properties of $\mathrm{Co}-\mathrm{Cr}-\mathrm{Mo}$ alloy are clearly revealed that its stress range is much larger than that of tool steel and its TMF life is twice as long as that of tool steel. The thickness loss of the TMF specimens, which is mainly attributed to the repeated spallation of oxide scale and re-oxidation of the exposed fresh alloy surface, are shown in Fig. 7 together with the corresponding rate of thickness loss. Obviously, thickness loss and especially the thickness loss rate of Co-Cr-Mo alloy are much lower than that of tool steel, indicating its excellent oxidation resistance in the present condition. In addition, the hardness of the specimens after TMF failure is compared with that before TMF test in Fig. 8. In contrast to the dramatic softening of tool steel, the Co-Cr-Mo alloy was obviously hardened during TMF test. In other words, the effects of thermomechanical cycles on the hardness of these two materials were completely opposite.

The above results indicate that the TMF properties of the wrought $\mathrm{Co}-\mathrm{Cr}-\mathrm{Mo}$ alloy were much superior to the tool steel under the test conditions, and the oxidation behavior and the hardness evolution were significantly different between two alloys during the TMF tests (Fig. 8). These phenomena are comparable with the previous researches of Birol. By investigating the thermal fatigue behavior of Co-based alloy, Ni-based alloy and tool steel, Birol [17-19] reported that Co-based alloy owned the highest resistance to thermal fatigue cracking, and more importantly, the high performance of Co-based alloy was ascribed to their high resistance to oxidation and temper softening. It was proved 
that the high temperature oxidation had significant effects on the initiation and propagation of cracks during TMF test. Neu et al. [22, 23] reported the TMF behavior of 1070 steel in the temperature range of $20-700{ }^{\circ} \mathrm{C}$, and the TMF life was 2-12 times longer in helium than in air. Nagesha et al. [24] also investigated the TMF behavior of $316 \mathrm{~L}(\mathrm{~N})$ stainless steel in the temperature range of $300-$ $650{ }^{\circ} \mathrm{C}$, and the TMF life in vacuum was six times as long as that in air. Therefore, the high temperature oxidation is an important damage mechanism in the TMF test $[22,23]$, and the superior TMF properties of $\mathrm{Co}-\mathrm{Cr}-\mathrm{Mo}$ in this study (Fig. 6) are partly benefit from its excellent oxidation resistance (Fig. 7). On the other hand, the mechanical properties of the materials in the corresponding temperature range of TMF tests also play an important role on the TMF performance $[11,25]$, so the high TMF properties of $\mathrm{Co}-\mathrm{Cr}-\mathrm{Mo}$ alloy also have a strong relationship with the high resistance to temper softening. Since the tool steel was mainly strengthened by the martensite, which is a non-equilibrium structure formed through quenching, the softening of the tool steel in this study was also widely observed in the tool steels operated at elevated temperature [7, 18, 25]. However, for the Co-Cr-Mo alloy, the increased hardness implies the structure evolution during TMF test.

The microstructure of the Co-Cr-Mo alloy after TMF failure is shown as EBSD maps in Fig. 9. By comparing with the initial structure in Fig. 5, the structure after TMF failure is remarkably different, indicating the significant effects of thermomechanical cycles on the microstructure. Owing to the $\gamma$-phase-to- $\varepsilon$-phase 
transformation, most of $\bigvee$ phase transformed into $\varepsilon$ phase. The $\varepsilon$ phase with an area fraction of $64.3 \%$ was observed distributed continuously while the $y$ phase was divided into the isolated zones (Fig. 9b). Compared with the initial average grain size of $12.1 \mu \mathrm{m}$ (Fig. 5), the $\varepsilon$ phase and the residual $\gamma$ phase after TMF failure owned the average grain size of $9.1 \mu \mathrm{m}$ and $13.7 \mu \mathrm{m}$, respectively. Moreover, the KAM map (Fig. 9c) indicated that the residual stress was obviously concentrated in the zone of $\varepsilon$ phase.

The final composite structure of $\mathrm{Y}$ phase with the fine-grained and stressconcentrated $\varepsilon$ phase is considered as the main reason of hardening of the Co$\mathrm{Cr}-\mathrm{Mo}$ alloy during TMF test [13]. According to the phase diagram of $\mathrm{Co}-\mathrm{Cr}-\mathrm{Mo}$ system [26], the $\varepsilon$ phase is more stable than the $\gamma$ phase at lower temperature, and the transformation temperature in the tested Co-Cr-Mo alloy is approximately $900{ }^{\circ} \mathrm{C}$. Therefore, the transformation from $\vee$ phase to $\varepsilon$ phase would be induced in Co-Cr-Mo alloy by the elevated temperature (lower than the transformation temperature) [26-28] or the mechanical strain [29-31]. Obviously, in the tested Co-Cr-Mo alloy, the $\varepsilon$ phase is more stable in the temperature range of TMF test and the $y$-phase-to- $\varepsilon$-phase transformation during TMF test was obviously observed in this study. However, the formation mechanism of the fine-grained and stress-concentrated $\varepsilon$ phase, in other words, how does the cyclic thermo-mechanical load affect the structure evolution in the $\mathrm{Co}-\mathrm{Cr}-\mathrm{Mo}$ alloy during TMF test, is still not clear. Therefore, the mechanism of the structure evolution in the tested Co-Cr-Mo alloy during TMF test should be clarified in the 
future. In addition, by comparing the TMF properties of different tool steels, Klobcar et al. [25] considered that the material with high strength and thermal stability at the corresponding temperature range would show a high resistance to TMF cracking, therefore, it is expected that a Co-based alloy with thermo-stable structure would exhibit higher resistance to TMF cracking.

\section{Conclusion}

In summary, under the simulated thermo-mechanical loading of die casting, the TMF life of the Co-Cr-Mo alloy was much longer than that of SKD61 tool steel. The higher TMF performance of the Co-Cr-Mo alloy was believed to be strongly related to its higher resistance to oxidation and tempering softening at elevated temperature. During the TMF test of the Co-Cr-Mo alloy, most of the initial $\mathrm{Y}$ phase transformed into the fine-grained and stress-concentrated $\varepsilon$ phase, and this structure evolution was considered to be responsible for the hardening of Co-Cr-Mo alloy.

\section{Acknowledgment}

This research was supported by a Regional Innovation Cluster Program 2010 from the Ministry of Education, Culture, Sports, Science and Technology of Japan and a Reconstruction Promotion Program (A-STEP) from Japan Science and Technology Agency. 


\section{Reference}

[1] D.R. Gunasegaram, M. Givord, R.G. O'Donnell, B.R. Finnin, Mater. Sci. Eng. A 559 (2013) 276-286.

[2] X. Sun, K.S. Choi, D.S. Li, Mater. Sci. Eng. A 572 (2013) 45-55.

[3] S. Shin, E. Kim, G. Yeom, J. Lee, Mater. Sci. Eng. A 532 (2012) 151-157.

[4] R. Song, S. Hanaki, M. Yamashita, H. Uchida, Mater. Sci. Eng. A 483-484 (2008) 343-345.

[5] Z.W. Chen, Mater. Sci. Eng. A 397 (2005) 356-369.

[6] B. Kosec, Metalurgija 47 (2008) 51-55.

[7] M. Muhi, J. Tu, F. Kosel, D. Klob, M. Pleterski, Metalurgija 49 (2010) 9-12.

[8] A. Persson, J. Bergstr, C. Burman, S. Hogmark, Surf. Coat. Tech. 146-147 (2001) $42-47$.

[9] P. Panjan, M. čekada, R. Kirn, M. Soković, Surf. Coat. Tech. 180-181 (2004) 561-565.

[10] K.S. Klimek, H. Ahn, I. Seebach, M. Wang, K.T. Rie, Surf. Coat. Tech. 174175 (2003) 677-680.

[11] A. Persson, S. Hogmark, J. Bergstr, Surf. Coat. Tech. 191 (2005) 216-227.

[12] F. Grube, E.E. Affeldt, H. Mughrabi, ASTM Special Technical Publication (2003) 164-179.

[13] J.R. Davis, ASM Specialty Handbook: Nickel, Cobalt, and Their Alloys, ASM International, Materials Park, 2000.

[14] A. Suzuki, T.M. Pollock, Acta Mater. 56 (2008) 1288-1297. 
[15] H.R. Lashgari, S. Zangeneh, F. Hasanabadi, M. Saghafi, Mater. Sci. Eng. A 527 (2010) 4082 - 4091.

[16] J. Mihelich, R.F. Kecker, Apparatus for processing corrosive molten metals, US Patent No. 5711366, 1998.

[17] Y. Birol, Mater. Sci. Eng. A 528 (2011) 1117-1124.

[18] Y. Birol, Mater. Sci. Eng. A 527 (2010) 1938-1945.

[19] Y. Birol, Mater. Sci. Eng. A 527 (2010) 6091-6097.

[20] N. Tang, Y.P. Li, S. Kurosu, H. Matsumoto, Y. Koizumi, A. Chiba, Corros. Sci. 53 (2011) 4324-4326.

[21] N. Tang, Y. Li, Y. Koizumi, S. Kurosu, A. Chiba, Corros. Sci. 75 (2013) 262 268.

[22] R.W. Neu, H. Sehitoglu, Metall. Trans. A 20 (1989) 1755-1767.

[23] R.W. Neu, H. Sehitoglu, Metall. Trans. A 20 (1989) 1769-1783.

[24] A. Nagesha, M. Valsan, R. Kannan, K.B.S. Rao, V. Bauer, H.J. Christ, V. Singh, Int. J. Fatigue 31 (2009) 636 - 643.

[25] D. Klobcar, J. Tusek, B. Taljat, Mater. Sci. Eng. A 472 (2008) 198-207.

[26] S. Kurosu, H. Matsumoto, A. Chiba, Metall. Mater. Trans. A 41 (2010) 26132625.

[27] H. Matsumoto, S. Kurosu, B. Lee, Y. Li, A. Chiba, Scripta Mater. 63 (2010) $1092-1095$.

[28] H. Matsumoto, Y. Koizumi, T. Ohashi, B. Lee, Y. Li, A. Chiba, Acta Mater. 64 (2014) 1 - 11. 
[29] A. Chiba, K. Kumagai, N. Nomura, S. Miyakawa, Acta Mater. 55 (2007) $1309-1318$.

[30] S. Kurosu, H. Matsumoto, A. Chiba, C. Landron, D. Fabregue, E. Maire, Scripta Mater. 64 (2011) 367 - 370.

[31] Y. Koizumi, S. Suzuki, K. Yamanaka, B. Lee, K. Sato, Y. Li, S. Kurosu, H. Matsumoto, A. Chiba, Acta Mater. 61 (2013) 1648 - 1661. 
Table 1

Chemical compositions of tested materials (wt. \%).

\begin{tabular}{cccccccccc}
\hline Alloy & Co & Fe & Cr & Mo & Si & Mn & V & N & C \\
\hline Co-Cr-Mo & Balance & 0.02 & 28 & 6 & 0.5 & 0.6 & -- & 0.12 & 0.04 \\
SKD61 & -- & Balance & 5 & 1.25 & 1 & $\leq 0.5$ & 1 & -- & 0.36 \\
* SKD61 is a tool steel grade in Japanese Industrial Standards (JIS), and its equivalent grade in American Society for \\
Testing and Materials (ASTM) is H13.
\end{tabular}




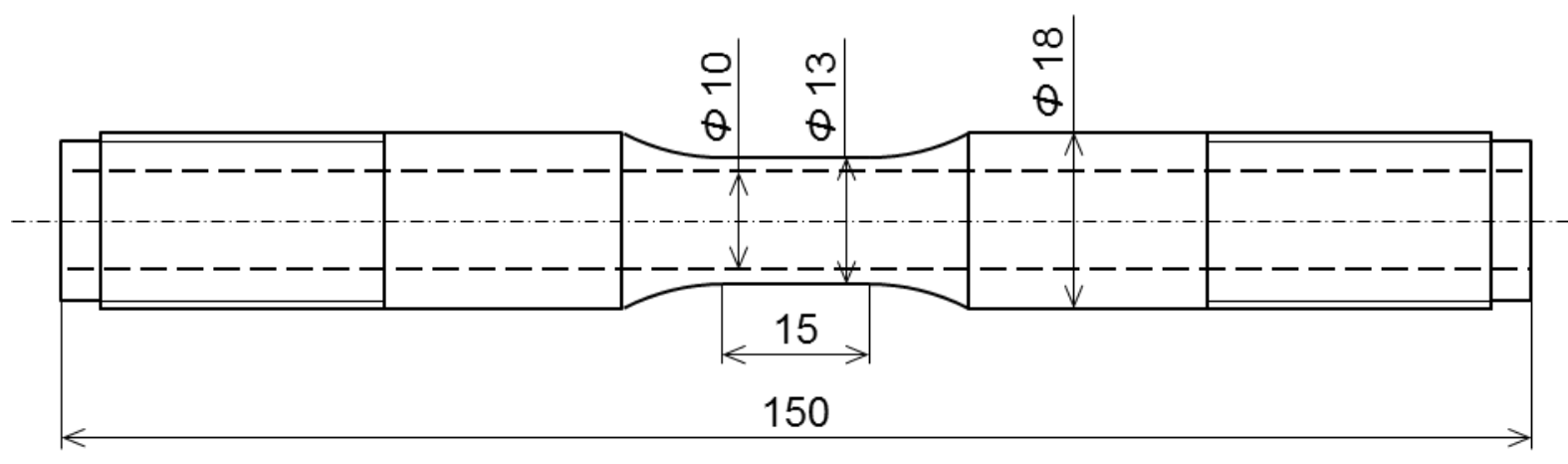

Fig. 1. The geometry of the tubular specimen. 
Cooling gas

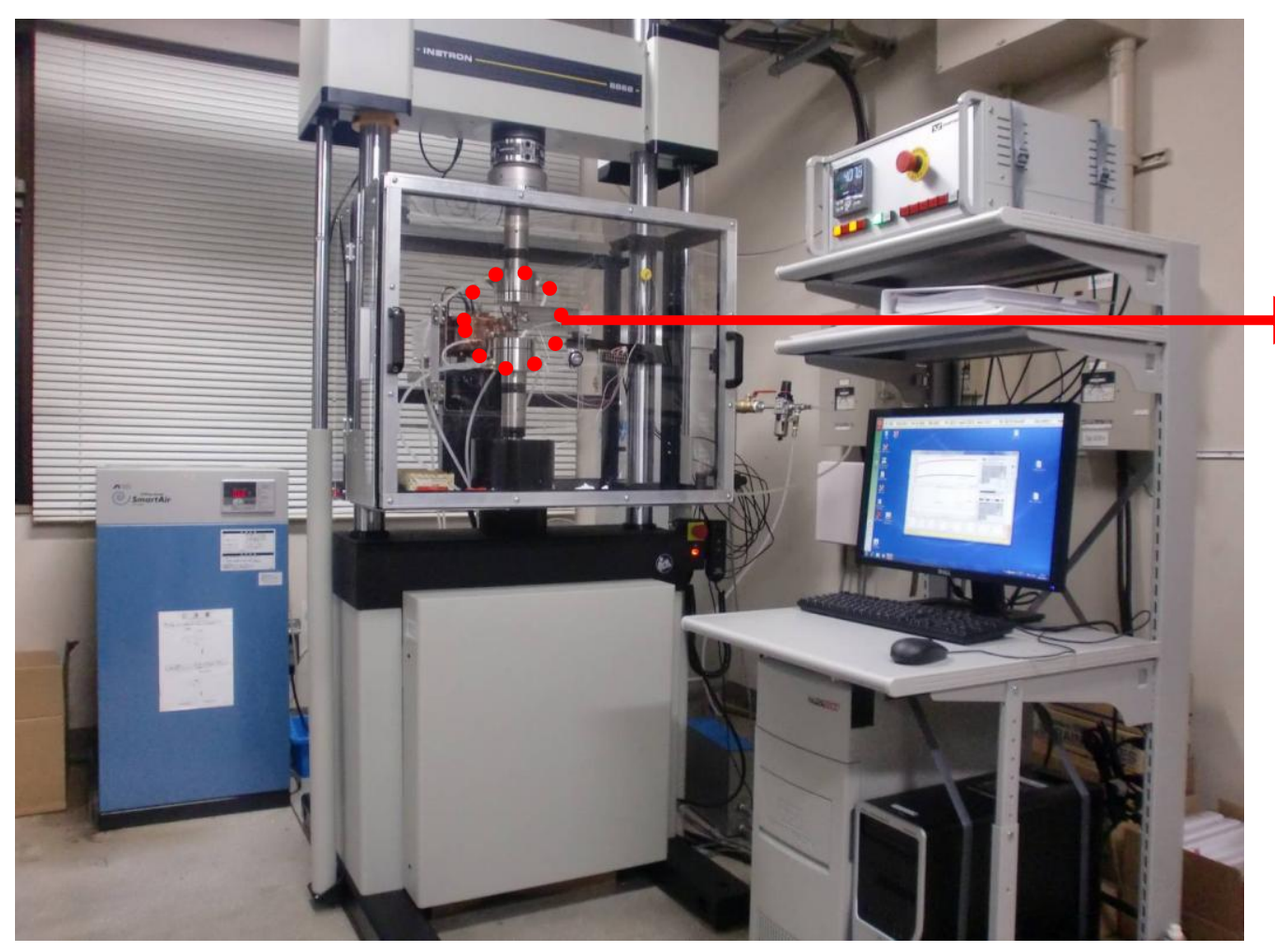

heating coil
Induction

(compressed air, 7-8 atm)

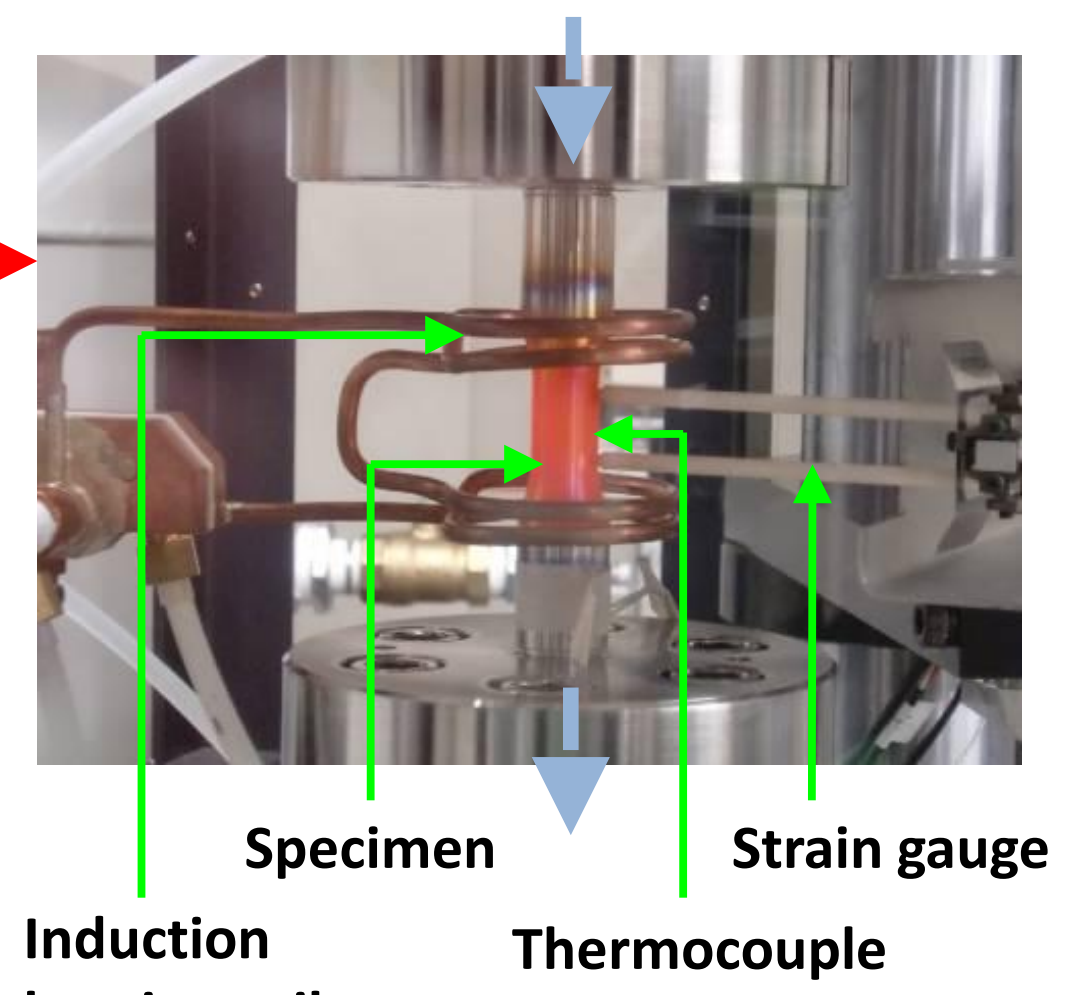

Thermocouple

Fig. 2. The Instron 8862 TMF tester. The specimen was heated with the induction heating coil and cooled with the compressed air. The temperature was monitored with a thermocouple and the strain was measured with a strain gauge. 


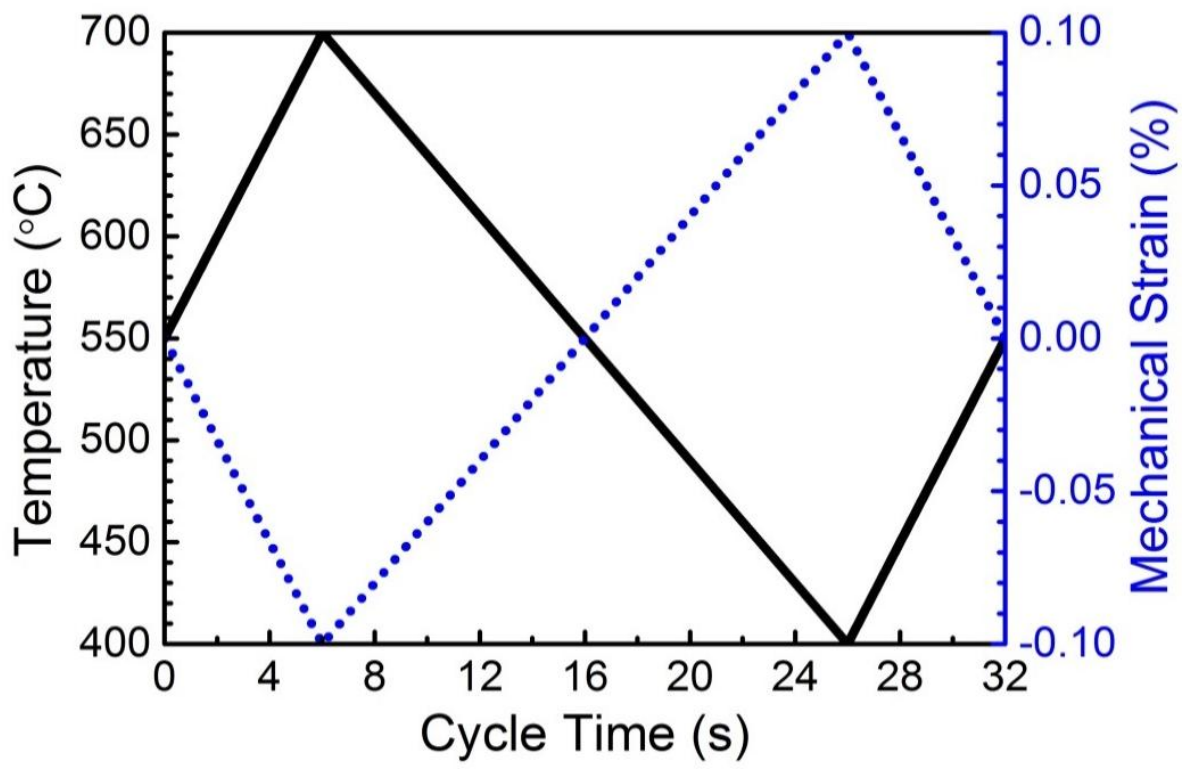

Fig. 3.The out-of-phase program with triangle waves of temperature and mechanical strain. 


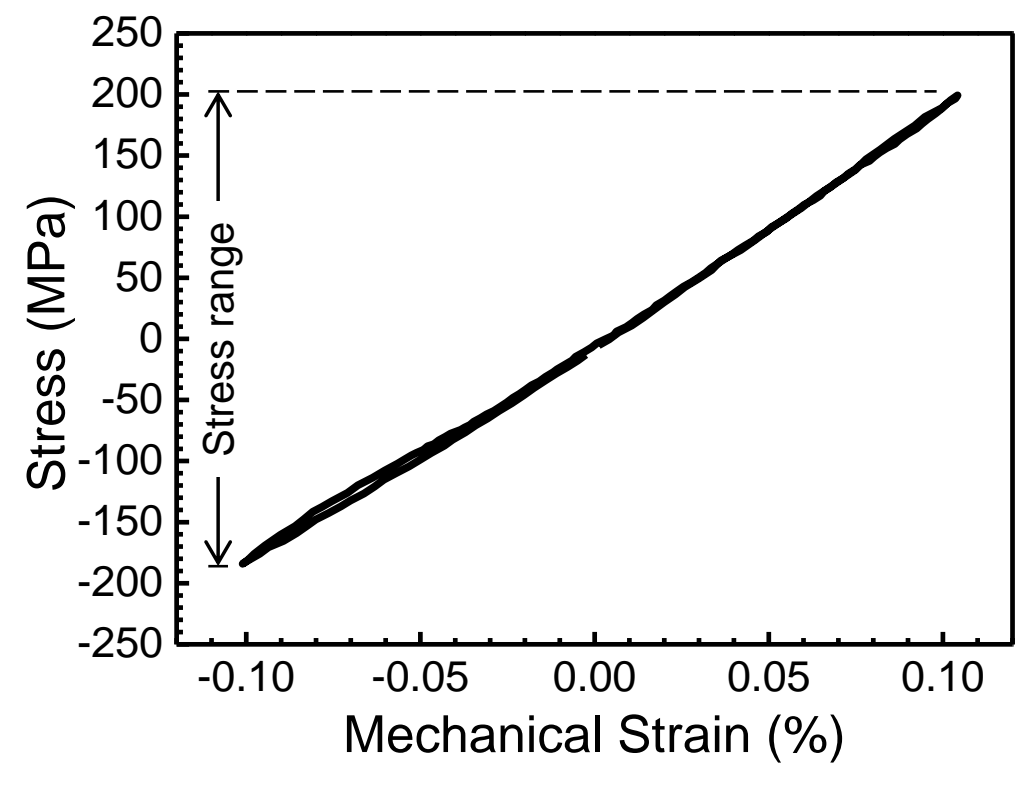

Fig. 4. Typical stress-strain loop of the Co-Cr-Mo alloy during TMF test. The stress range (as indicated) of each TMF cycle was recorded by the tester automatically. 

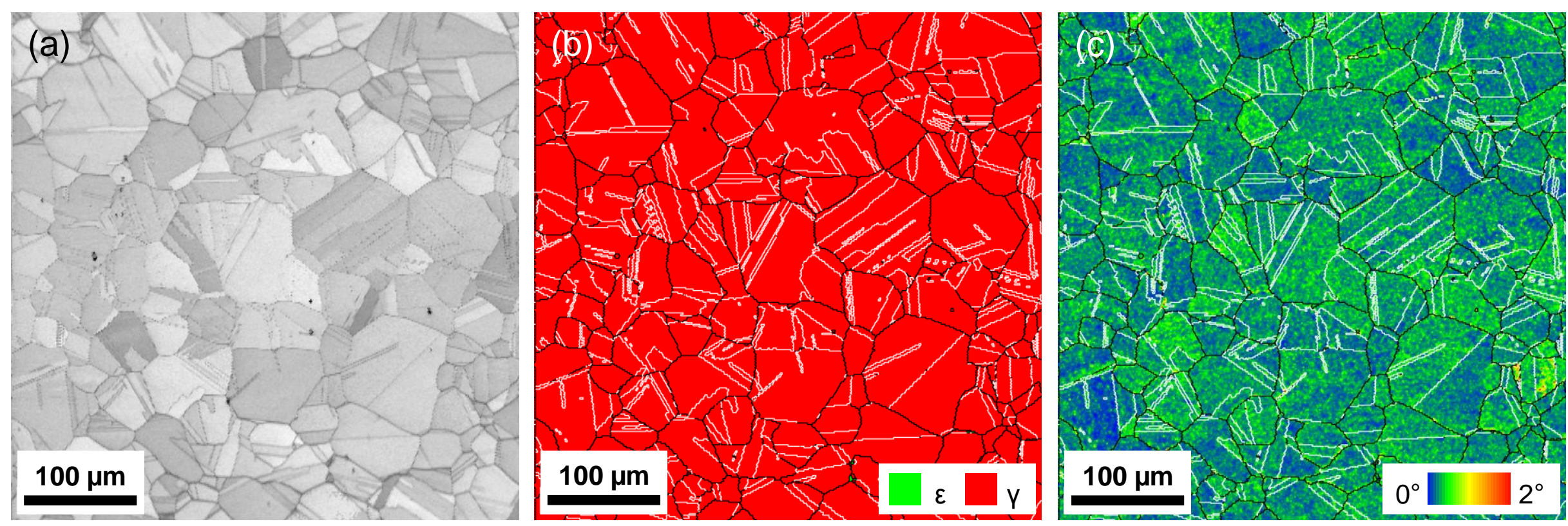

Fig. 5. EBSD maps of the Co-Cr-Mo alloy before TMF test. (a) IQ map. (b) Phase map and (c) KAM map with high angle boundaries (black line) and $\Sigma 3$ twin boundaries (white line). 


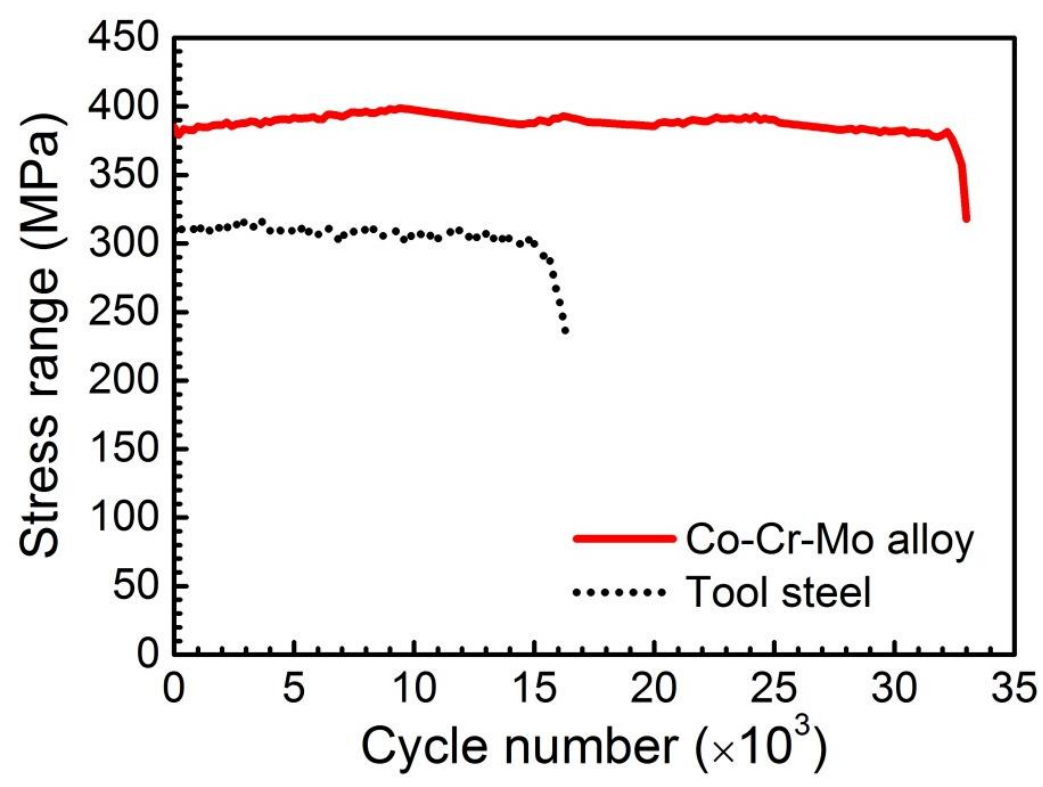

Fig. 6. Stress range of the Co-Cr-Mo alloy and tool steel as a function of cycle number. In comparison with tool steel, the $\mathrm{Co}-\mathrm{Cr}-\mathrm{Mo}$ alloy showed larger stress range and longer TMF life. 


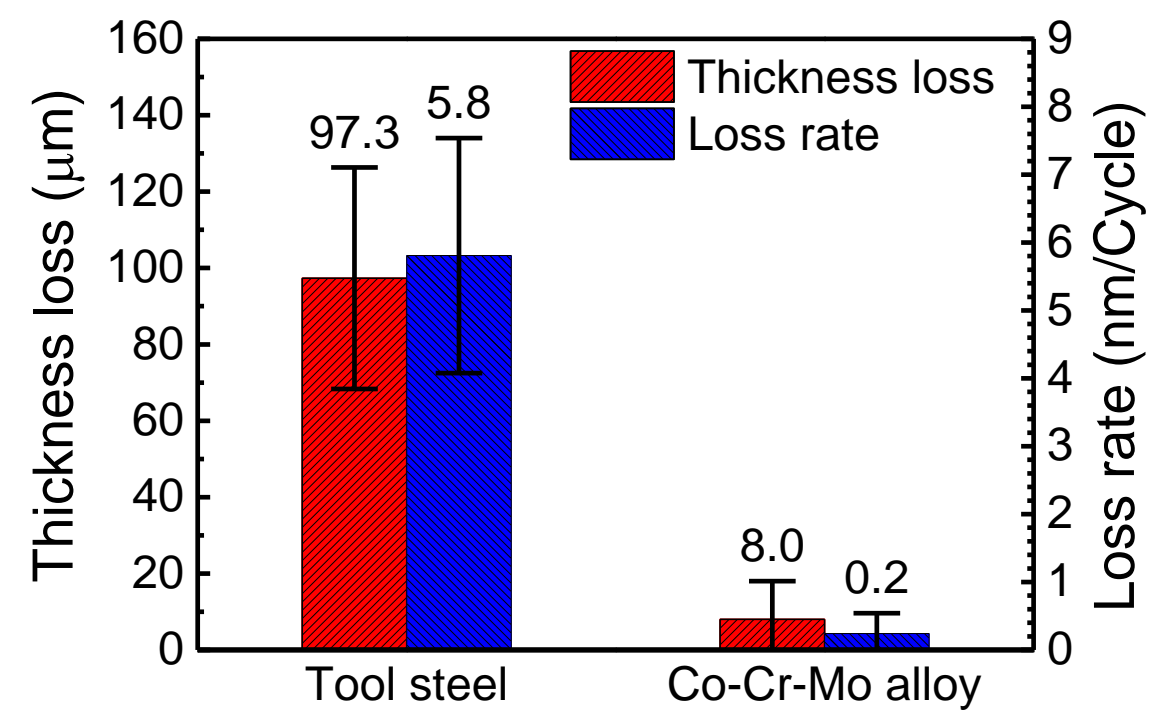

Fig. 7. Thickness loss of the specimens after TMF failure and the average rate of thickness loss during TMF tests. In comparison with tool steel, the Co-Cr-Mo alloy showed higher oxidation resistance. 


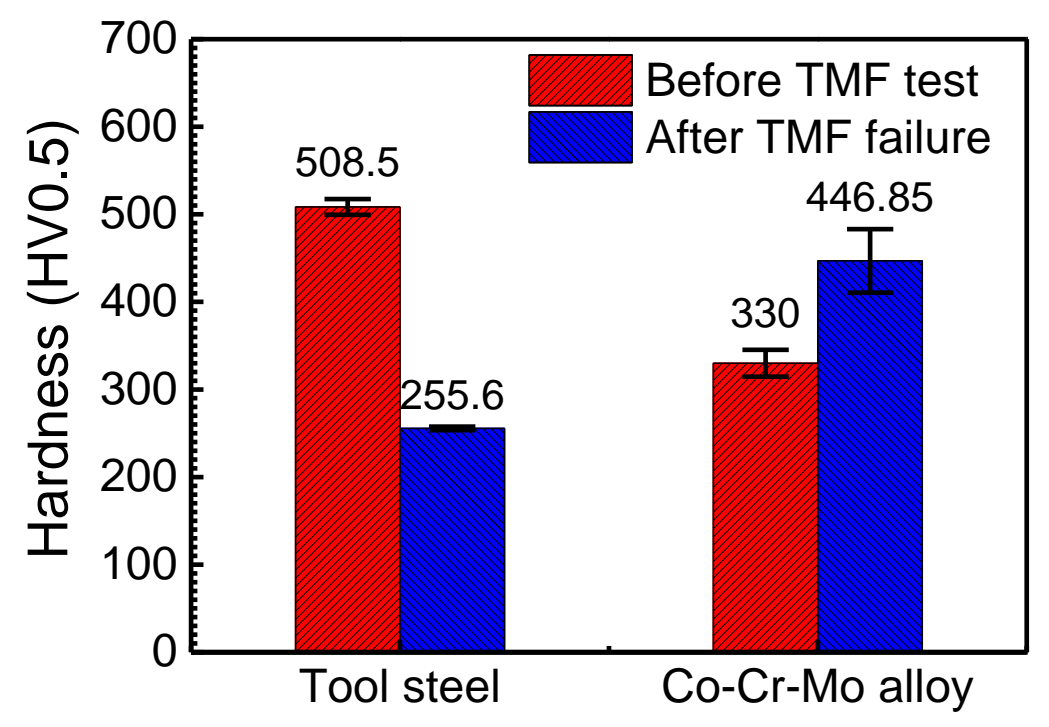

Fig. 8. Hardness of the two alloys before TMF tests and after TMF failure. The hardness of Co-Cr-Mo alloy significantly increased after TMF test in contrast to the softening of tool steel. 

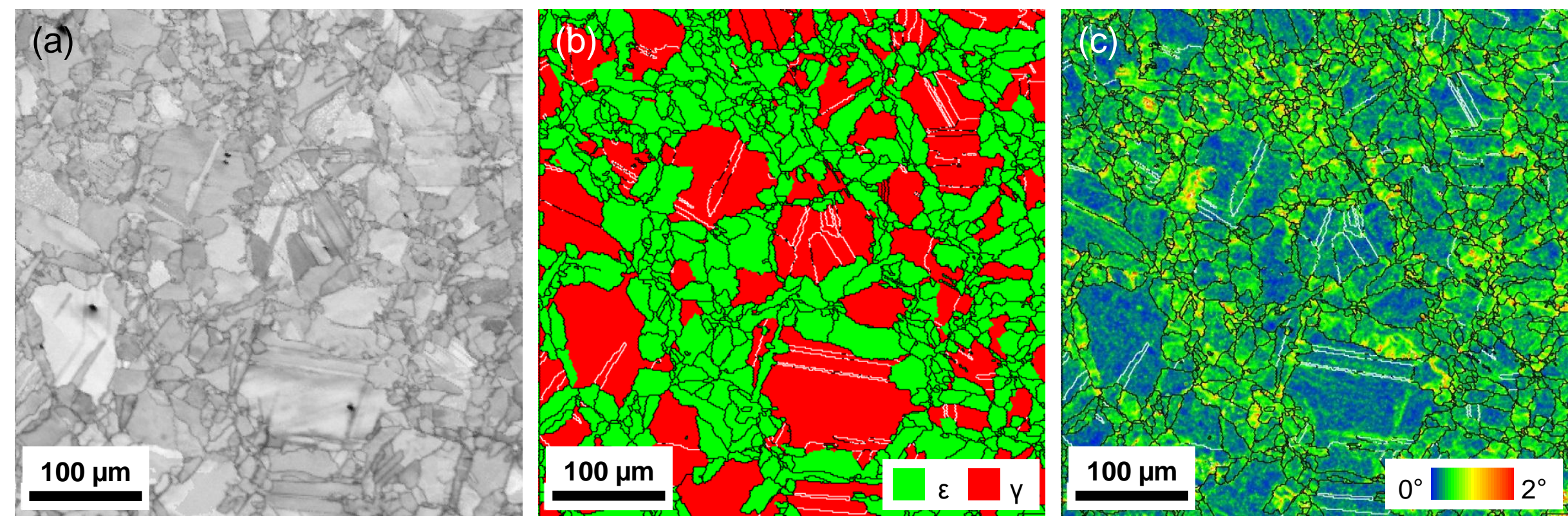

Fig. 9. EBSD maps of the Co-Cr-Mo alloy after TMF failure. (a) IQ map. (b) Phase map and (c) KAM map with high angle boundaries (black line) and $\Sigma 3$ twin boundaries (white line). 